Escuela de Relaciones Internacionales.

Universidad Nacional, Costa Rica.

N. ${ }^{\circ} 90.1$ • Enero-Junio de 2017

doi: http://dx.doi.org/10.15359/ri.90-1.3

Pp. $87-100$

\title{
EL MODELO DE FRANQUICIA COMO ESTRATEGIA PARA EL POSICIONAMIENTO DE LAS PYMES DEL SECTOR RESTAURANT-BAR EN SINALOA
}

\author{
THE FRANCHINE MODEL AS POSITIONING STRATEGY \\ OF SMES IN RESTUARANT- BAR SECTOR IN SINALOA
}

\section{Myrna Cristina Morales Avila*}

\section{RESUMEN:}

La presente investigación se realizó en la ciudad de Culiacán, Sinaloa, durante el periodo comprendido de 2015 a 2016, su objeto de estudio son las pequeñas y medianas empresas (PYMES) del giro restaurant-bar; compara el modelo de negocios que utilizan las empresas del mismo sector con modelos de franquicias, esto es, para resolver la problemática a la que se afronta este tipo de negocios en cuanto a las fallas operativas con las que se manejan y a su poca permanencia en el mercado en el que se desarrollan.

Palabras clave: PYMES, restaurant-bar, modelo de negocios, franquicia, Sinaloa.

\begin{abstract}
:
This research was conducted in the city of $\mathrm{Cu}-$ liacán, Sinaloa, during the period from 2015 to 2016, taking as an object of study small and medium enterprises (SMEs) Whirl restaurant- bar and comparing the business model used by entrepreneurs in the same industry with franchise models, that is, to solve the problem to which this business is facing in terms of operational faults which already handle their short stay in the market in which they develop.
\end{abstract}

Keywords: SMEs, restaurant- bar, business model, franchise, Sinaloa.

* Licenciada. Dirección General de Investigación y Posgrado. Facultad de Contaduría y Administración. Maestría en Administración Estratégica. Énfasis en Dirección de Negocios Internacionales. Universidad Autónoma de Sinaloa. México. Correo electrónico: myrnamorales@uas.edu.mx 


\section{Análisis del Contexto}

\section{Entorno global}

Las pequeñas y medianas empresas (PYMES) son de suma importancia, no solo en México, sino en todo el mundo, ya que concentran las dos terceras partes de empleo total mundial, según un estudio de Ayyagari (2011), el cual presenta información de 99 países correspondiente al período 2006-2010, lo que lo convierte en el estudio más completo realizado hasta la fecha.

La definición del término PYME suele variar de un país a otro $\mathrm{y}$, a menudo, se basa en el número de personal trabajador, el volumen de negocios anual o el valor de los activos de la empresa. Se entiende por microempresa toda organización que ocupa hasta diez trabajadores o trabajadoras; las pequeñas empresas son aquellas que cuentan con un personal constituido por 10 y 100 individuos y las medianas, aquellas que cuentan con una plantilla de entre 100 y 250 personas, es decir, se entiende por PYME toda empresa que ocupa a menos de 250 personas (OIT, 2015).

El 30 de diciembre de 2009, la Secretaría de Economía publicó, en el Diario Oficial de la Federación (DOF), una estratificación con los criterios que aparecen en la tabla 1:

\section{Tabla 1}

Clasificación por sector según el número de personal empleado

\begin{tabular}{|l|c|c|c|}
\hline \multicolumn{4}{|c|}{ Sector } \\
\hline \multicolumn{4}{|c|}{ Clasificación según el número de empleados } \\
\hline Tamaño & Industria & Comercio & Servicios \\
\hline Micro & De 0 a 10 & De 0 a 10 & De 0 a 10 \\
\hline Pequeña & De 11 a 50 & De 11 a 30 & De 11 a 50 \\
\hline Mediana $A$ & De 51 a 250 & De 31 a 100 & De 51 a 100 \\
\hline
\end{tabular}

Nota: Elaboración propia, con datos del DOF (septiembre de 2009).

Según la Organización Internacional del Trabajo OIT (2015), se estima que, en todo el mundo, existen entre 420 y 510 millones de PYMES; de estas, el 9 por ciento pertenece a la economía formal (sin contar las microempresas) y entre el 80 y el 95 por ciento están situadas en países de ingresos bajos y medianos. 
Por otra parte, según datos de INEGI (2015), en México existen cerca de 17000 empresas del giro restaurant-bar, giro al cual se enfocará el presente trabajo de investigación; de este número se desprenden en la zona del Estado de Sinaloa cerca de 600, de las cuales 250 se encuentran en el municipio de Culiacán, en el cual se desarrollará este estudio.

La categoría restaurant-bar se sitúa como una de las favoritas entre los grupos emprendedores de la región, por ser una de las más rentables y mantenerse en constante crecimiento. Según reporta la Cámara Nacional de la Industria Restaurantera y Alimentos Condimentados (Canirac), en México existen unos 428,000 restaurantes, que mueven cerca de \$182,992 millones de ventas anuales y representan el $1.4 \%$ del Producto Interno Bruto (PIB) nacional (Entrepreneur, 2014).

En el sector de las franquicias mexicanas, este segmento ocupa el primer lugar del giro de alimentos y bebidas en cuanto a número de empresas (con 63 marcas o el 16\% del total), de acuerdo con el directorio 500 Franquicias de Entrepreneur. Además, hay que tener en cuenta aquellos restaurantes especializados en determinados tipos de gastronomía (ubicados en categorías como comida japonesa, saludable, taquerías, etc.) (Entrepreneur, 2014). Así, pues, se observan algunos casos exitosos como los que presenta el modelo de Starbucks ${ }^{1}$, que arribó a México en 2002 y ya cuenta con más de 400 sucursales en el país (El Financiero, 2014) y El Fogoncito, empresa mexicana que lleva más de 30 años en el mercado y la cual cuenta con sucursales en Costa Rica y Honduras; ambas marcas han demostrado tener un amplio conocimiento en el área de las franquicias, estudiando su funcionamiento y procedimientos.

\section{Problemática actual}

El progreso económico y social de un país y, por ende, de sus habitantes, descansa en la creación continua de nuevas empresas y en el crecimiento y la consolidación de las existentes. Las micro, pequeñas y medianas empresas (MIPYMES) tienen particular importancia, no solo por sus aportaciones a la producción y distribución de bienes y servicios, sino también por el gran potencial de generación de empleos, lo que representa el medio ideal para distribuir la riqueza y el bienestar. Asimismo, según datos de los Censos económicos de INEGI (2015), los servicios de hotelería y restaurantes son de los sectores que mayor aportación hacen al valor agregado, los cuales fueron de $12.5 \%$ en 2008

1 El modelo Starbucks presenta, entre sus características más destacadas y de éxito, el ofrecer a los clientes su establecimiento como un "tercer lugar", invertir en su personal con capacitaciones constantes y tratarlo como socio y no como empleado, y la atención a los detalles, entre otras (Michelli, 2007). 
y de $11.5 \%$ en 2013 ; cabe destacar el porcentaje de personal ocupado que cubren: de $25.4 \%$ en 2008 y de 25.3 en 2013. Resalta, así, la importancia que tiene para la economía del país.

Lamentablemente, en nuestro país estas empresas no viven, más bien sobreviven (SISC, 2015). Dolan (2004) enuncia a continuación los principales problemas que enfrentan las pequeñas y medianas empresas:

- Falta de sistemas de calidad y mejora continua

- Sin su misión y visión de la empresa bien definida

- Tecnología de producción inadecuada

- Información de gestión insuficiente

- Productividad insuficiente

- Estructuras organizativas inadecuadas

- Falta de garantías para acceder al crédito

- Recursos humanos poco calificados

- Poca atención a los mercados internacionales

- Estructuras financieras inadecuadas

También, es importante agregar que, según la investigación demográfica económica presentada por INEGI (2015), los negocios más pequeños, que constituyen el 95\% de las empresas en el país, son los más volátiles, y que la esperanza de vida tiene un comportamiento creciente conforme aumenta la edad de los negocios.

Así, de acuerdo a la Asociación Nacional de Franquicias (2012), en México el $60 \%$ de los negocios independientes (los que no son franquicias) no completan el segundo año de vida, a diferencia de las franquicias, de las cuales el 95\% siguen vivos al quinto año de vida.

Lo que para Frías (2012) confirma que existen diversas razones, pero las principales son: la experiencia en el negocio, los procesos asentados y una marca 
conocida. Cuando se adquiere una franquicia, en realidad se está comprando un modelo de negocio que ya funciona, con capacitación incluida, es decir, que aprenderá como hacer cada una de las cosas necesarias para llevar a cabo el negocio (procesos) y con una marca que la gente ya identifica y que se hace publicidad de diversas maneras. Esto, aunque no elimina por completo los riesgos de un fracaso, disminuye considerablemente sus probabilidades de fracasar, como lo reflejan las cifras antes mencionadas.

\section{Planteamiento del problema}

Según Zazueta (2015), las PYMES del sector restaurant-bar presentan carencias en cuanto a la implementación de un sistema organizacional y de liderazgo efectivo o falta de delegación y comunicación por parte de los altos rangos hacía el personal que trabaja en horario operativo, desde ahí comienzan a observarse las fallas e insuficiencias a las que los empleados y empleadas tienen que someterse y, en consecuencia, la escasez de satisfacción de la clientela, o bien, al desconocimiento y falta de capacitación de parte de la empresa hacia los sujetos empleados del horario operativo en cuanto a los procesos, servicios, productos y promociones que se pretende implementar; muchas veces, estos se ven en la necesidad de inventar los propios para que la empresa se mantenga a flote a falta de manuales operativos, procedimientos y del entrenamiento correspondiente para que todo se realice de forma correcta en la empresa, es decir, es necesaria la estandarización de procedimientos para que una empresa de este tipo de giro funcione correctamente.

Asimismo, el presidente de la Asociación Mexicana de Restaurantes, Francisco Mijares (2013), asevera que el principal reto de quienes desean integrarse al sector "es la falta de conocimiento de cómo debe manejarse un restaurante y la sobrerregulación de sus operaciones, por lo que a los dos años de operación, sólo dos de cada 10 restaurantes que abren empresarios que no son restauranteros, sobreviven".

Las alternativas para la solución de problemas que enfrentan las PYMES se basarán más que nada en los modelos de franquicia o cadenas de empresas de servicio, en las maneras en que se administran y las formas en las que manejan a su personal, para, así, poder implementarlos en las empresas de la localidad para asegurar su permanencia en el mercado. 


\section{Formulación del problema de investigación}

Las PYMES del sector restaurant-bar de la localidad no cuentan con un modelo de negocios adecuado que permita su permanencia en el mercado, desembocando así en un limitado tiempo de vida y, como consecuencia, en la pérdida de las inversiones de los grupos pequeños empresarios y emprendedores, causando despido de personal, grandes reinversiones por la necesidad de estarse reinventando constantemente a falta de una identidad propia y una marca que permanezca en la mente de los consumidores.

\section{Objetivo general}

El objetivo general de la presente investigación es el de recabar datos bibliográficos que nos ayuden a entender la situación de las PYMES del sector restaurant-bar y la necesidad de crear un modelo de negocio que les ayude a permanecer en el mercado, tomando como ejemplo el de las franquicias.

\section{Justificación de la investigación}

En cuanto a la justificación, esta investigación está orientada a tratar de demostrar que las pequeñas y medianas empresas (PYMES) del giro restaurant-bar se enfrentan a diferentes problemáticas, en cuanto a procesos de sistematización, suministro, operaciones y cadena de mando. Esto desemboca en su poca permanencia en el mercado en el que operan. Este artículo compara el modelo de negocios que utilizan las empresas del mismo sector con modelos de franquicias, los cuales han probado ser una de las mejores técnicas para emprender y permanecer en el mercado.

El propósito del presente trabajo de investigación es crear un modelo que pueda ser utilizado por los grupos emprendedores en las PYMES del sector restaurant-bar en Culiacán, Sinaloa, para asegurar un mejor desempeño y poder darles una pauta en cuanto a procesos de sistematización y cadenas de mando, suministro y operaciones.

Las actividades que se realizan en las empresas deben estar orientadas de tal manera que todas las acciones que se lleven a cabo estén enfocadas en obtener mejor eficiencia con respecto a los procesos, servicios, cadena de mando y suministros, así como al liderazgo estratégico y su importancia en la toma de decisiones en las PYMES del sector antes mencionado. 
Los resultados que se obtengan de este trabajo servirán de guía para orientar a los grupos empresarios dedicados a la actividad de restaurant-bar.

\section{Marco teórico referencial}

\section{Antecedentes investigativos}

El Centro Asia-Pacífico para la Excelencia en Franquicias de la Griffith University, en Australia, realizó un estudio, entre 2010 y 2012, acerca del éxito y fracaso de PYMES, tanto dentro de un sistema de franquicias como fuera. Este estudio se centró en la forma en que los negocios lidian con tiempos económicos difíciles y los factores que pueden haber contribuido a su supervivencia (Serralta, 2013).

El informe reveló que tanto negocios franquiciados como pequeños negocios independientes se vieron afectados por la crisis económica de forma parecida, y que los factores principales que afectaban el éxito de una forma más crítica se referían a la manera en que cada persona gestionaba su situación. Por lo tanto, la personalidad de cada empresa afectaba en gran medida a su éxito, tanto sus niveles de motivación como su voluntad y habilidad para adaptarse. Tener más libertad para reconocer las circunstancias cambiantes y actuar en consecuencia permite a los grupos empresarios adaptarse a las presiones o cambios económicos, lo cual les es muy beneficioso (Serralta, 2013).

A continuación, se encontró el estudio de Solano (2014) con el título "Visión del emprendedor hacia las franquicias", en el cual se utilizó la investigación cualitativa para la recolección de datos relevantes para posteriormente dar apertura a su selección y análisis. Asimismo, dentro de los datos seleccionados se presentó el alcance descriptivo, a fin de describir las situaciones, fenómenos y eventos que se suscitaron dentro del sistema de franquicias, con el único propósito de evidenciar los perfiles y características más sobresalientes.

Las conclusiones a las que se llegaron en este trabajo de investigación son que para comprender mejor el modelo de franquicias, lo más conveniente es contar con toda la información referente al tema de interés, bien lo dijo Jorge Valencia López, quien es director general de la consultaría inter franquicias "La mejor estrategia para ingresar a la red de franquicias es contar con tres aspectos importantes y estos son: conocimiento, conocimiento y conocimiento" (Solano, 2014).

Asimismo, se encontró el análisis de May, Aguilera y Loy (2011), con el título Aportación e importancia de las franquicias en la economía mexicana: 
¿Realidad o fantasía?, en el cual se puede asumir que la metodología que se utilizó también fue cualitativa, ya que, la recolección de datos relevantes fue importante para después analizarlos. Además, con los datos recabados se presentó el alcance descriptivo, a fin de detallar el funcionamiento del modelo de franquicias.

Las resoluciones a las que se llegaron en el estudio de May et al. (2011) son las siguientes: no cabe duda alguna de que la franquicia se ha consolidado como la modalidad de desarrollo empresarial de mayor crecimiento en el mundo. $\mathrm{Su}$ presencia en los diferentes países presenta diferentes grados de desarrollo, según el nivel de aceptación y características de los grupos emprendedores nacionales a los que se haga referencia. Es tal la importancia del formato que actualmente existen más de 17 mil redes de franquicias y más de un millón de puntos de venta y servicios entre franquiciados y puntos propios pertenecientes a estas redes. Podría parecer que solamente en Estados Unidos, Europa y Latinoamérica se ha venido dando este fenómeno, pero lo increíble es que los asiáticos lo han adoptado de manera tan buena que China hoy, con sus cientos de millones de habitantes, es el primer lugar en la materia, según datos del World Franchise Council (año).

\section{Conceptos y evolución histórica del modelo de franquicia}

De acuerdo con el diccionario de la Real Academia de la Lengua Española, encontramos que franquicia es "la libertad y exención que se concede a una persona para no pagar derechos por las mercancías que introduce o extrae, o por el aprovechamiento de algún servicio público en particular", otra definición dice que "es un sistema de venta de productos de una firma comercial en una tienda de otro propietario y bajo ciertas condiciones económicas", cualquier que sea la definición que se adopte, la franquicia para términos del presente trabajo es, de acuerdo con May et al. (2011): un formato de negocio con posicionamiento de marca que permite la explotación de un concepto desarrollado y probado en su viabilidad y documentado en manuales por un ente emprendedor denominado franquiciante a través del otorgamiento de los derechos a otro ente emprendedor inversionista denominado franquiciatario, cuya unión o alianza se estipula en las cláusulas de un contrato de franquicia.

\section{Primeros sistemas de franquicia}

El primer antecedente de franquicia se ubica en los Estados Unidos, probablemente con el otorgamiento a particulares por vía legislativa para la explotación 
de algunos servicios o "public utilities" como fue el caso de los ferrocarriles y los bancos. Durante la década de 1850 a 1860, Singer Sewing Machine Company atravesaba por serios problemas para la distribución de sus productos en el país. En principio decidió cobrar a sus vendedores en vez de pagarles para crear un esquema de concesionarios mediante el cual compraban una máquina para revenderla con un sobreprecio; la empresa resolvió cambiar su estructura básica de funcionamiento estableciendo una red de concesionarios a quienes se les cobraba una participación por el derecho a distribuir sus máquinas de coser en territorios específicos, esto debido a los altos costos laborales que implicaba el sostenimiento de numeroso personal vendedor directo. Singer mantuvo este esquema solo por 10 años, estableciéndose como una de las marcas más reconocidas en el territorio norteamericano y sembrando así las bases del actual sistema de franquicias (Chavarría, 2007).

Los primeros casos de franquicia en México se dieron a conocer cuando McDonald's decidió entrar en el país en 1985 aprovechando los cambios que se estaban suscitando en el país, tales como la nacionalización de la banca, lo cual impuso un decreto en el control de cambios en el país y se suspendió el pago de la deuda externa al registrarse los índices de reserva interna más bajos de la historia. En este escenario la empresa sienta las bases para su ingreso al mercado mexicano y se convierte en una de los pioneras del sistema, seguida de Kentucky Fried Chicken, Howard Johnson, Bing, Holanda, Holiday Inn, Pizza Hut, entre otras (Chavarría, 2007).

Actualmente, de acuerdo con Forbes (2016), en México existen poco más de 1,200 marcas de franquicias; según cifras de la AMF, 74\% son de origen nacional. No obstante, muy pocas franquicias mexicanas se pueden jactar de poseer el valor de mercado y la presencia a nivel local y mundial como el que tienen sus pares de Estados Unidos, convertidas en las marcas de franquicias más codiciadas en el mundo.

Dicho lo anterior, está ampliamente documentado que las empresas mexicanas, en particular el segmento de micro y pequeñas, han tenido que sortear un número de problemas por las condiciones que el entorno de negocios les impone; en los últimos años la caída de la demanda es uno de los factores más decisivos. Otros factores más estructurales son el acceso restringido a fuentes de financiamiento; bajos niveles de capacitación de los recursos humanos y escaso acceso a innovación y tecnología (SISC, 2015).

En el caso particular de Sinaloa, el presidente de CANIRAC señala que se estima que en la capital el número aproximado de restaurantes que cierra cada año es de tres a cuatro (2014). 


\section{Marco teórico}

Este trabajo de investigación se sustenta en las teorías principales en las que se desarrolla una empresa, tomando como referencia fundamental las teorías clásicas de la administración del estadounidense Frederick Winlow Taylor (1911), el cual desarrolló la llamada escuela de administración científica, preocupada por aumentar la eficiencia de la industria a través, inicialmente, de la racionalización del trabajo operario; así como también del europeo Henry Fayol (1916), quien desarrolló la llamada teoría científica preocupada por aumentar la eficiencia de la empresa a través de su organización y de la aplicación de principios generales de la administración con bases científicas. Las ideas de estos dos autores constituyen las bases del llamado enfoque clásico tradicional de la administración, cuyos postulados dominaron aproximadamente las cuatro primeras décadas de este siglo en el panorama administrativo de las organizaciones (Romero, 2015).

Caben destacar también los trabajos de estudio realizados por Frank y Lilian Gilbreth (1900), quienes estudiaron la relación de tiempos y movimientos, es decir, el desarrollo del mejor método de trabajo que permita al personal ejecutar las tareas en el menor tiempo, con mayor facilidad y satisfacción, así como también se enfocaron en los aspectos humanos y psicológicos de los grupos trabajadores comprendiendo sus necesidades y permitiendo así que las tareas se proyectaran de manera que su ejecución requiriera el gasto y la tensión física mínima (Romero 2015).

También se recogieron como referencia principal tanto la teoría organizacional, de la cual se tomará como fuente principal a Idalberto Chiavenato (2006), el cual nos dice que el campo del conocimiento humano que se encarga del estudio de las organizaciones en general y a medida que estas crecen y se desarrollan, necesitan de la administración; así como también a Hodge, Anthony y Gales (2007), los cuales nos hablan de la estructura, lo cual definen como la suma total de las formas en las que una organización divide su mano de obra en tareas diferentes y su posterior coordinación.

Cabe destacar la importancia de las teorías del desarrollo organizacional, las cuales, De Faria Mello (2006) define como un nuevo arte de perfeccionar la organización, integrando necesidades de la empresa y necesidades de las personas; una nueva filosofía de administración; una nueva manera de administrar, día tras día, un estilo administrativo renovador y revitalizador; una nueva tecnología; un conjunto práctico de instrumentos y técnicas para cambios planeados, en que los aspectos psicológicos y de comportamiento deben recibir la debida atención. Chiavenato (2006), por su parte, también menciona el cambio 
organizacional planeado, el cual es un enfoque de sistema abierto y propone un cambio de la cultura de la organización.

Es importante señalar la teoría de la ventaja competitiva que propone Michael Eugene Porter (1985), la cual guarda una relación estricta con el concepto de valor o la cadena de valor, la cual no es otra cosa que un modelo teórico que grafica y permite describir las actividades de una organización para generar valor al cliente final y a la misma empresa; el mismo autor menciona también que:

La ventaja competitiva crece fundamentalmente en razón del valor que una empresa es capaz de generar. El concepto de valor representa lo que los compradores están dispuestos a pagar, y el crecimiento de este valor a un nivel superior se debe a la capacidad de ofrecen precios más bajos en relación a los competidores por beneficios equivalentes o proporcionar beneficios únicos en el mercado que puedan compensar los precios más elevados. ... Una empresa se considera rentable si el valor que es capaz de generar es más elevado de los costos ocasionados por la creación del producto (Porter, 1985).

También es de suma importancia agregar la teoría de la estrategia. En ese sentido, es importante señalar que han sido diversos estudios los que han tratado de definir el concepto de estrategia, entre los más destacados se observan las aportaciones de Neumann y Morgenstem (1944) -en su obra sobre la teoría del juego-, quienes definieron la estrategia empresarial como la serie de actos que ejecuta una empresa, los cuales son seleccionados de acuerdo con una situación concreta. Así mismo, Drucker (1954), en su libro The Practice of Management, propuso que las gerencias deben analizar la situación actual y futura de la organización para poder plantear una estrategia adecuada.

Por último, pero no menos importante, se señalan las teorías de internacionalización, las cuales, para el objetivo del énfasis en dirección de negocios internacionales, en la presente maestría en administración estratégica, cuya inclusión es fundamental en nuestro estudio como base para fundamentarlo. Las teorías de la internalización estudian los procesos internos de transferencia de información en las empresas, tratando de explicar por qué las transacciones de productos intermedios, sean tangibles o intangibles -know how-, entre países están organizadas por jerarquías, en lugar de estar determinadas por las fuerzas del mercado, es decir, su planteamiento central afirma que las organizaciones multinacionales son un mecanismo alternativo al mercado para gestionar actividades de valor a través de fronteras nacionales. Según Root (1994) y Rialp (1999), la internacionalización es un conjunto de operaciones que facilita el establecimiento de vínculos más o menos estables entre la empresa y los mercados internacionales, a lo largo de un proceso de creciente implicación y 
proyección internacional. Por su parte, Johanson y Vahlne (1977) la describen como un proceso en el cual las empresas incrementan gradualmente su nivel de actuación en los mercados internacionales.

\section{Conclusiones}

Se vuelve necesaria la incorporación de un modelo de negocios que permita a estas organizaciones permanecer en el mercado y ser competitivas. En ese sentido, se propone el modelo de franquicia como una alternativa de solución para que el restaurant-bar incorpore estrategias de liderazgo, administración, manejo de personal, capacitación, optimización de los recursos y herramientas de trabajo, etc., que permitan resolver, en el corto plazo, la problemática descrita, y que estos negocios permanezcan de manera exitosa en el mercado que operan.

El desarrollo e implementación de un plan de negocios basado en el modelo de franquicias permite al sector restaurant-bar lograr una posición competitiva en el mercado de Culiacán Sinaloa, a partir de la mejora en sus manuales de operación, sistematización y procedimientos, así como lograr un liderazgo organizacional.

En resumen, se recomienda ampliamente el modelo de franquicia como estrategia de posicionamiento en el mercado sinaloense, dado que es un modelo de éxito probado por diversas empresas nacionales e internacionales del sector restaurant-bar, es decir que, si las PYMES de la localidad toman como ejemplo a seguir un modelo de franquicia y lo adaptan a su empresa para operar, su probabilidad de permanencia en el mercado será mayor a la de la media nacional. 


\section{Referencias}

Ayyagari, M., Demirgüç-Kunt, A. y Maksimovic, V. (2011). Small vs. young firms across the world: Contribution to employment, job creation, and growth. World Bank Policy Research Working Paper No. 5631.

Chavarría López, Leticia R. (2007). Las Franquicias como una opción de crecimiento para las microempresas: Propuesta de un modelo. México, D.F.: Instituto Politécnico Nacional. Escuela Superior de Comercio y Administración.

Garduño, Sergio (2015) Life \& Death of SME's. Consultores Internacionales, S.C. No. 697. México, D.F.

Soto, E.(2004). Las Pymes ante el desafio del siglo XXI. México, D.F.: Thomson learning.

Druker, Peter. (1954). The Practice of Management. New York. Business Harper and Row. Entrepreneur. (2014). 10 franquicias de restaurantes y bares. Recuperado de https://www.entrepreneur.com/article/267578

Frías, Angélica. (2012). Ideas para PyMES. El modelo de franquicias: ganando más con menores riesgos. Recuperado de http://www.ideasparapymes.com/franquicias/modelo franquicias financiamiento aumentando retorno inversion.html

Forbes. (2016). Las 15 mejores franquicias en México. Recuperado de http://www. forbes.com.mx/las-15-mejores-franquicias-en-mexico/

Instituto Nacional de Estadística y Geografía (INEGI). (2015). Número de empresas del giro Restaurant-bar. Recuperado de http://www3.inegi.org.mx/ sistemas/mapa/denue

Johanson y Vahlne (1977). The Internationalization Process of the Firm-A Model of Knowledge Development and Increasing Foreign Market Commitments. Journal of International Business Studies (JIBS) 8, 23-32. doi: 10.1057/palgrave.jibs. 8490676

May Hernández, F., Aguilera Muñoz, O., Loy Enriquez, R. (2011). Aportación e importancia de las franquicias en la economía mexicana: ¿Realidad o fantasía? En Global Conference on Business and Finance Proceedings, 6(2). The Instiute of Business and Financial Reseach. San José, Costa Rica.

Michelli, Joseph A. (2007). The Starbucks experience, 5 Principles for Turning Ordinary Into Extraordinary. New York: McGraw-Hill. Organización Mundial de Trabajo. (2015). IV informe. Autor.

Porter, Michael Eugene. (1985). Competitive Advantage. Strategic Management. 
Rialp Criado, Alex. (1999). Los enfoques micro-organizativos de la internacionalización de la empresa: Una revisión y síntesis de la literatura. Revista ICE, 1. Recuperado de http://www.revistasice.info/cachepdf/ICE $781 \quad 117128 \quad 5$ F6320F1F9F499454DA33296 EB4ECF86.pdf

Romero M, y Beatriz,(2015). Cambio organizacional para el desarrollo exportador en la mediana empresa hortícola del estado de Sinaloa (Tesis nivel Doctorado). Universidad de Occidente. México.

Serralta de Colsa, Ana. (2013). Un estudio revela porqué los franquiciados tienen éxito en épocas de crisis. Franquicia Directa. España. Recuperado de http:// www.franquiciadirecta.com/blog/unestudiorevelaporquelosfranquiciadostienenexitoenepocasdecrisis/? $\mathrm{r}=2563$

Solano Nava, Adriana. (2014). Visión del emprendedor hacia las franquicias.

Universidad Autónoma del Estado de Zumpango, Estado de México.

Universidad Autónoma del Estado de Zumpango, Estado de México. Vivavoz. (2014). Estadística de Cierre de Restaurantes Continúa y Puede Incrementarse. Recuperado de http://www.vivavoz.com.mx/voz-urbana-menu/10001estadistica-de-cierre-de-restaurantes-continua-igual-y-puede-incrementar$\underline{\text { se-canirac }}$

Zazueta Valencia, Vladimir. (2015). Satisfacción laboral y satisfacción de los comensales en un restaurante sinaloense. Facultad de Contaduría y Administración. Maestría en Administración Estratégica. Universidad Autónoma de Sinaloa. 